\title{
Diabetes and lower extremity amputation - rehabilitation pathways and outcomes at a regional hospital
}

\author{
P Manickum, ${ }^{1}$ (D) SS Ramklass, ${ }^{1}$ (iD) TE Madiba $^{2}$ (D) \\ ${ }^{1}$ School of Clinical Medicine, College of Health Sciences, University of KwaZulu-Natal, South Africa \\ ${ }^{2}$ Department of General Surgery, School of Clinical Medicine, College of Health Sciences, University of KwaZulu-Natal, South Africa
}

Corresponding author, email: prabashni1@gmail.com

Background: Lower extremity amputations (LEAs) are most frequently due to diabetes mellitus (DM), a disease on the rise. The objective of this study was to determine the prevalence and aetiology of LEAs at Addington Hospital from 2013 to 2017 and to explore the physiotherapy referral practices and outcomes.

Methods: Retrospective study carried out at Addington Hospital, Durban. Patients who underwent LEAs were filtered from theatre registers and the hospital Meditech database. Data collected included patients' demographic profile, diabetic status, level of amputation, limb orientation, physiotherapy referral status, and rehabilitation outcomes. Physiotherapy files were scanned for the attendance of referred patients. Study endpoints were prevalence, diabetes status, referral status, compliance and rehabilitation outcomes.

Results: From 2013 to 2017,1028 LEAs in 843 patients were identified with single amputations (697) and multiple amputations (146). The median age was 61 (IQR 52-68) years, and the M:F ratio was 1.3:1. A total of 574 (68.1\%) patients had DM. Seven hundred and thirty-eight (71.8\%) amputations were as a result of DM. The level of amputations was below-knee $(479 ; 46.6 \%)$, toectomy $(236 ; 23 \%)$, above-knee $(196 ; 19 \%)$ and trans-metatarsal $(117 ; 11.4 \%)$. Only 148 patients $(17.6 \%)$ were referred for physiotherapy, of which $91(61.5 \%)$ attended. Mobility in those who attended rehabilitation was with a walking frame $(51 ; 56 \%)$, crutches $(29 ; 31.9 \%)$, prosthesis and crutches $(7 ; 7.7 \%)$, and wheelchair-bound $(4 ; 4.4 \%)$.

Conclusion: Over half the amputations were associated with DM, which was also a risk factor for multiple amputations. Although referral and attendance for physiotherapy were very poor, mobility in those who attended was excellent, indicating a dire need to improve hospital referral pathways.

Keywords: prevalence, diabetes mellitus, lower extremity amputations, rehabilitation, physiotherapy

\section{Introduction}

Indications for major limb amputation include diabetic foot sepsis, peripheral vascular disease (PVD), trauma, and neoplasms. ${ }^{1}$ The prevalence of lower extremity amputation (LEA) incidence varies across the world, ${ }^{2}$ and the global amputation rates range between 3.8 and 23.6 per $10^{5} .^{3}$ The prevalence of the various indications shows marked geographical variation, with varying differences in prevalence between high-income countries (HICs) and lowand middle-income countries (LMICs). ${ }^{4}$ This variation in amputation rates may be explained by multiple background factors such as differences in disease burden, comorbidities, socioeconomic status and other economic factors. ${ }^{2,4}$

Amputations of the lower limb are devastating events that pose psychological (amputees are often perceived or perceive themselves as "incomplete" individuals) and financial burden on patients and their families, as well as the considerable cost to healthcare providers. ${ }^{5-8}$ Rather than being the end of care, an amputation actually becomes the beginning of care since patients who undergo LEAs often endure lengthy hospital stays from morbidity and initial rehabilitation, ${ }^{1}$ followed by life-long care and follow-up in order to address rehabilitation and inherent comorbidities. ${ }^{9}$

A recent study has reported on lower limb amputations below the knee from the same institution. ${ }^{10}$ However, the study was limited to amputations below the knee. In order to improve on that limitation, the authors undertook the current study which reports on amputations on all levels in the lower extremities. With this paper, we wish to establish if the observations on amputations below the knee can be generalised to amputations at all levels.

Against this background, we conducted a retrospective analysis of amputees over a five-year period at a regional hospital in Durban to determine the number of all LEAs and the referral practices for rehabilitation and outcomes.

\section{Materials and methods}

This was a descriptive retrospective chart review conducted on all patients presenting with LEAs attending Addington Hospital from 1 January 2013 to 31 December 2017. Addington Hospital is a regional hospital based in the eThekwini district of the KwaZulu-Natal Province in South Africa. The referral system for Addington Hospital includes 


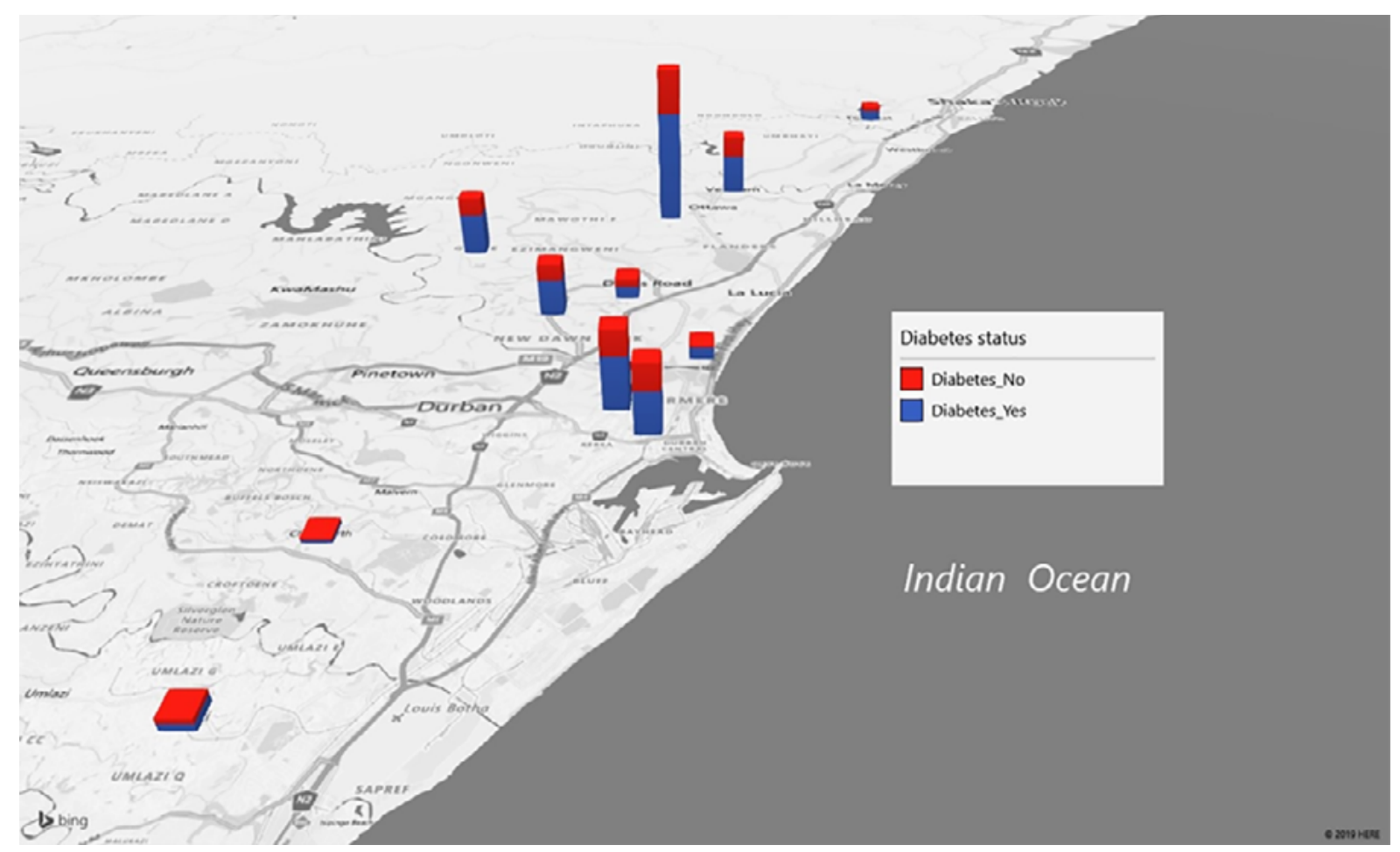

Figure 1: Geographical map of number of patients stratified according to area of residence

clinics and district hospitals situated in the northern part of Durban, as shown in Figure 1, a geospatial map of patients referred to Addington Hospital for amputations. According to the criteria used by the South African Government, population groups are defined as African, Indian, Coloured, and White. ${ }^{11}$ In South Africa, "Coloured" refers to people of mixed ancestry. ${ }^{12}$ Amputations were performed by surgeons, and patients were selectively referred to the physiotherapy clinic for rehabilitation. The hospital has the capacity to offer the rehabilitation services of physiotherapists, orthotists, prosthetists, psychologists and social workers.

Purposive sampling was used and all patients who presented with LEAs during the study period were included. A data collection sheet was used to collect the following data: patient's age, gender, race, area of residence, diabetic status, year of amputation, level of amputation, referral status to physiotherapy, number of physiotherapy visits and rehabilitation outcome. Data were obtained from theatre registers; patients' hospital numbers were then scanned through the hospital Meditech database for referral status to physiotherapy, number of physiotherapy visits, and any additional information not available in the theatre registers. Physiotherapy records retained in the physiotherapy department were used to confirm physiotherapy visits and rehabilitation outcomes up to their last visit. The frequency of visits was allocated a 'zero' frequency if patients failed to comply with physiotherapy after referral. Among the patients who did present for physiotherapy, the frequency of physiotherapy visits was classified into categories of 10 visits.

The amputation levels were defined as follows: abovethe-knee amputations (AKA) involve removal of the leg from the body by cutting through both the thigh tissue and femoral bone; below-knee amputation (BKA) is a trans-tibial amputation that involves removing the foot, ankle joint, and distal tibia and fibula with related soft tissue structures; trans-metatarsal amputation (TMA) is a weight-bearing amputation at the mid-tarsal level preserving the additional length of the foot; and a toe amputation involves removal of a single or multiple toes at the level of the metatarsalphalangeal joints. ${ }^{13,14}$

Data collection commenced following regulatory approvals for the study from the Biomedical Research Ethics Committee of the University of KwaZulu-Natal (BREC 236/19), the provincial Department of Health (KZ_201909_034), the hospital manager, physiotherapy manager, and the theatre unit manager.

The results of this study were analysed after being coded and transferred to a Microsoft Excel spreadsheet. Data were analysed using the Statistical Package for Social Sciences (SPSS version 22). Categorical variables were summarised using proportions/percentages and presented graphically using bar charts or tables, while continuous variables were summarised using means (standard deviations). Comparisons of various categories were tested using Pearson's chi-square test. If an expected cell count had fewer than five observations, then Fisher's exact test was employed. Adjustment for multiple testing (using the Bonferroni correction) was employed. An adjusted $p$-value of $<0.05$ was considered statistically significant.

\section{Results}

The derivation of the study sample is shown in Figure 2. Out of the 909 patients identified, 66 entries were excluded due to incorrect patient file numbers or omission of data from theatre registers and the Meditech database (Figure 2). Eight hundred and forty-three patients had LEAs, of whom 574 $(68 \%)$ had diabetes mellitus (DM). Six hundred and ninetyseven patients had a single amputation, and 146 patients had more than one amputation. Eight hundred and forty-three patients underwent 1028 amputations.

The profile and characteristics of patients undergoing amputations are listed in Table I. Males predominated among both diabetic and non-diabetic patients. Left-sided 
Table I: Profile of patients with lower extremity amputations and amputation parameters

\begin{tabular}{lccc}
\hline Characteristic & Total & Diabetic & Non-diabetic \\
\hline Total patients & 843 & $574(68.1 \%)$ & $269(31.9 \%)$ \\
Demographics & & & $59(46-67)$ \\
\hline Median age (IQR) & $61(52-68)$ & $61.5(53-69)$ & $166: 103$ \\
Male:Female & $474: 369$ & $308: 266$ & $1.6: 1$ \\
M:F ratio & $1.3: 1$ & $1.2: 1$ & - \\
Left:Right limb & $438(52 \%): 405(48 \%)$ & - & 269 \\
Number of amputations & & & 21 \\
\hline One amputation & 843 & 574 & 0 \\
Two amputations & 146 & 125 & 0 \\
Three amputations & 31 & 31 & 0 \\
Four amputations & 6 & 6 & 290 \\
Five amputations & 2 & 2 & 738 \\
Total amputations & 1028 & & \\
Level of amputation & & 360 & 119 \\
\hline Below-knee & 479 & 149 & 47 \\
Above-knee & 196 & 145 & 91 \\
Toes & 236 & 84 & 33 \\
Trans-metatarsal & 117 & &
\end{tabular}

* In patients with multiple amputations, subsequent amputations were performed on the same leg

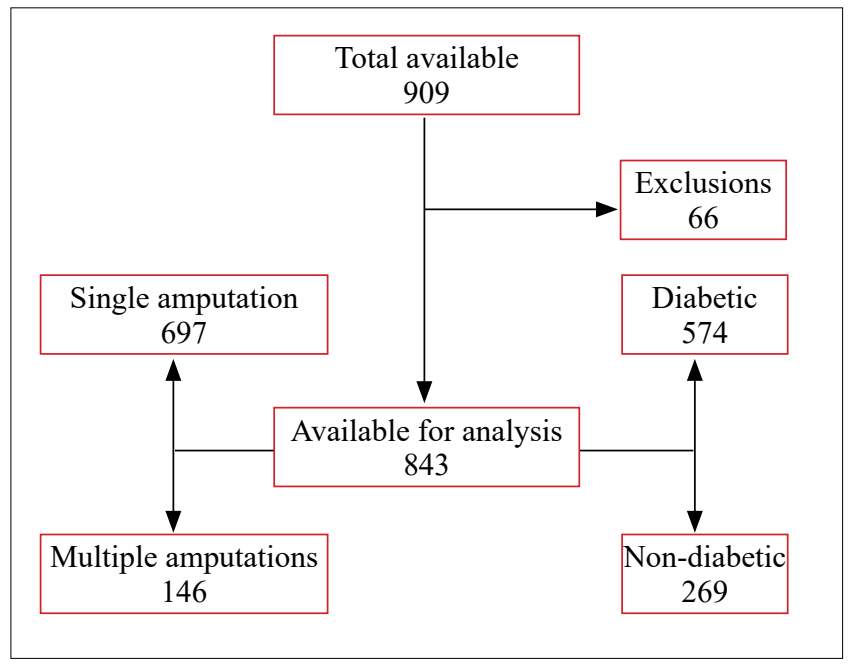

Figure 2: Snapshot of number of amputations

amputations were slightly more common than right-sided amputations. More than half of the patients in this study had DM $(68 \%)$, with more than half $(738 ; 71.8 \%)$ of the amputations being due to DM. One hundred and twentyfive $(16.9 \%)$ diabetic patients and $21(7.2 \%)$ non-diabetic patients had more than one amputation $(<0.001)$. Among patients with multiple amputations, non-diabetic patients had a maximum of two amputations, whereas amputations ranged between two and five among diabetic patients. Four hundred and seventy-nine amputations were BKAs (46.6\%), 236 were toe amputations (23\%), 196 were AKAs (19\%), and 117 were TMAs $(11.4 \%)$. This trend was seen in both diabetic and non-diabetic amputees, although diabetic amputees predominated across the board. There were 398 African patients (260 diabetic; 138 non-diabetic), 376 Indian patients (271 diabetic; 105 non-diabetic), 31 White patients (20 diabetic; 11 non-diabetic), 31 Coloured patients

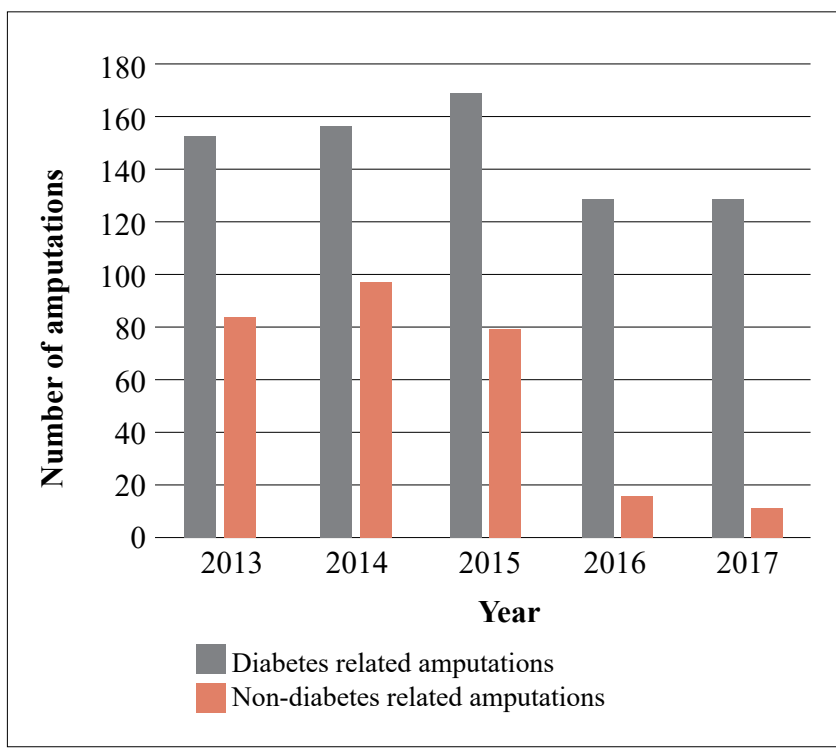

Figure 3: The number of amputations per year from 2013-2017

(18 diabetic; 13 non-diabetic) and seven foreign patients (five diabetic; two non-diabetic).

Diabetic amputees were greater than non-diabetic amputees in every category (Figure 1). The largest number of patients with amputations was from the Phoenix area (250; $29.7 \%$ ) followed by Central Durban (102; 12.1\%), Verulam $(99,11.7 \%)$, Inanda $(91 ; 10.8 \%)$, Newlands $(80 ; 9.5 \%)$ and Overport $(60 ; 7.1 \%)$.

Figure 3 illustrates the number of amputations that were performed per year. Diabetes-related amputations (71.8\%) remained high over the five years but peaked in 2015 . Non-diabetic amputations (28.2\%) showed a reduction in numbers over the last two years of the study. 


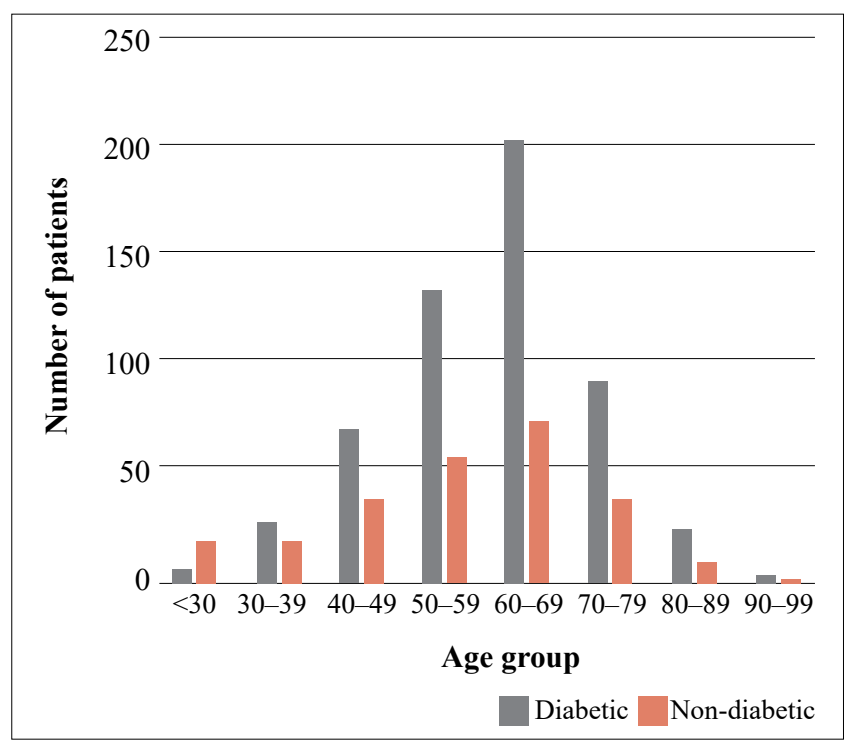

Figure 4: Profile of diabetic and non-diabetic lower extremity amputees stratified according to age groups

The median age for diabetic and non-diabetic patients was 61.5 (53-69) and 59 (46-67) years, respectively. Figure 4 shows the number of amputees stratified according to age. The peak ages for both diabetic and non-diabetic patients were in the seventh decade. A higher number of diabetesrelated amputations was observed in all age groups except those under 30 years, where non-diabetes-related amputations predominated $(p=0.006)$.

One hundred and forty-eight of the 843 patients with amputations (17.6\%) were referred for physiotherapy (108 diabetics and 40 non-diabetics). Only 91 of these patients attended and, among those that did attend, 54 patients presented for $\leq 10$ physiotherapy sessions (41 diabetic; 13 non-diabetic), 30 attended $11-20$ sessions (24 diabetic; six non-diabetic), and seven attended $>20$ sessions (four diabetic; three non-diabetic). Sixty-nine diabetic amputees and 22 non-diabetic amputees attended physiotherapy (Table II). The majority of the patients $(95.6 \%)$ who attended physiotherapy were mobile with either a walking frame or crutches. There was no significant difference in mobility outcomes between diabetic and non-diabetic patients $(p=0.055)$.

\section{Discussion}

This study has identified several findings on patients presenting with LEA. There was a male preponderance, and diabetic patients were only slightly older than non-diabetic patients. In the international literature, LEA incidence is gender-related, with higher rates in males, ${ }^{15}$ which is similar to the findings in our study. This is in contrast to a recent South African study which showed a female preponderance among lower extremity amputees. ${ }^{16}$ The incidence of LEA increases with age. ${ }^{15}$ In the current study, diabetic and non-diabetic patients presented with amputations at a younger age (median 61 years) compared to the 67 years reported in a Canadian study by Kayssi et al. ${ }^{17}$ In the recent South African study by Mtshali et al., patients with LEAs presented at a mean age of 60.95 years, ${ }^{16}$ thus suggesting that South African amputees tend to be younger than in the international literature. Data from other LMICs reported age at presentation ranging between 48 and 69 years for all amputations. ${ }^{7,18,19}$ This has important implications related

Table II: Mobility outcome in 91 patients who attended physiotherapy

\begin{tabular}{|c|c|c|c|}
\hline Mobility according to level of amputation & $\begin{array}{c}\text { Diabetic patients } \\
n(\%) \\
\end{array}$ & $\begin{array}{c}\text { Non-diabetic patients } \\
n(\%) \\
\end{array}$ & Total \\
\hline \multicolumn{4}{|l|}{ AKA } \\
\hline Total & 11 & 4 & 15 \\
\hline Crutches & $3(27.3 \%)$ & $4(100 \%)$ & $7(46.7 \%)$ \\
\hline Walking frame & $7(63.6 \%)$ & 0 & $7(46.7 \%)$ \\
\hline Wheelchair & $1(9.1 \%)$ & 0 & $1(6.7 \%)$ \\
\hline \multicolumn{4}{|l|}{ BKA } \\
\hline Total & 42 & 13 & 55 \\
\hline Crutches & $11(26.2 \%)$ & $6(46.2 \%)$ & $17(30.9 \%)$ \\
\hline Crutches and prosthesis & $2(4.8 \%)$ & $3(23.1 \%)$ & $5(9.1 \%)$ \\
\hline Walking frame & $27(64.3 \%)$ & $4(30.8 \%)$ & $31(56.4 \%)$ \\
\hline Wheelchair & $2(4.8 \%)$ & 0 & $2(3.6 \%)$ \\
\hline \multicolumn{4}{|l|}{ TMA } \\
\hline Total & 4 & 1 & 5 \\
\hline Crutches & $2(50 \%)$ & $1(100 \%)$ & $3(60 \%)$ \\
\hline Crutches and prosthesis & $1(25 \%)$ & 0 & $1(20 \%)$ \\
\hline Walking frame & $1(25 \%)$ & 0 & $1(20 \%)$ \\
\hline \multicolumn{4}{|l|}{ Toes } \\
\hline Total & 12 & 4 & 16 \\
\hline Crutches & $2(16.7 \%)$ & 0 & $2(12.5 \%)$ \\
\hline Crutches and prosthesis & $1(8.3 \%)$ & 0 & $1(6.3 \%)$ \\
\hline Walking frame & $8(66.7 \%)$ & $4(100 \%)$ & $12(75 \%)$ \\
\hline Wheelchair & $1(8.3 \%)$ & 0 & $1(6.3 \%)$ \\
\hline Grand total & 69 & 22 & 91 \\
\hline
\end{tabular}

* 87/91 were mobile with crutches, prosthesis or walking frame; 7/91 were fitted with a prosthesis 
to socioeconomic burden because of the early presentation of disability in the person's life. Variation in the causes of amputations may be explained by multiple background factors such as surgical decision-making, differential health delivery systems and general healthcare infrastructure. ${ }^{4,20}$ Other patient-related factors include differences in disease burden and comorbidities, socioeconomic status, and other economic factors. ${ }^{4,20}$ Major amputations are more frequent in countries with lower GDP per capita and healthcare expenditures, with several studies from the African setting reporting amputations for trauma as the main indication. . $^{1,415,20}$

More than two-thirds of the amputations were attributed to diabetes. The increasing etiological role of diabetes leading to amputations was seen throughout the five years of the study. International studies have shown similar trends with diabetes-related amputations predominating over nondiabetic amputations..$^{7,8,17-19}$ The majority of diabetic-related amputations are preceded by foot ulcers which act as a portal for infection leading to sepsis or gangrene that may require amputation..$^{21,22}$ This is further compounded in diabetics by peripheral vasculopathy and neuropathy which also contribute to the risk of amputation. ${ }^{23}$ There is geographical variation in amputation rates worldwide. ${ }^{4,20,24}$ Trauma is the predominant cause in India, parts of the United States and parts of Africa. ${ }^{20,24}$ Several studies have highlighted DM as an important cause of amputations. ${ }^{8,17,19}$ Indeed, a previous local study ${ }^{10}$ also showed a higher proportion of diabetesrelated amputations below the knee, although the figure was lower than in the present study (53\% vs $68 \%$ ).

More males than females underwent LEAs in this series. This is consistent with global literature, which reports a male preponderance. ${ }^{7,18,24}$ The laterality of LEAs has drawn conflicting results in the literature. There were more left-sided amputations in this series. Other studies have demonstrated a preponderance of right-sided amputations. ${ }^{10,18,25}$ Coxon and Gallen postulated that the laterality of amputations could be due to limb dominance. Right or left footedness governs the limb used most often for initiating or ending movement. ${ }^{25}$ Hence, the dominant limb may be exposed to more shearing or mechanical trauma, predisposing it to more stresses and resultant injury. ${ }^{25,26}$

There is variability in the prevalence of amputation levels in the lower extremity among different studies. BKAs predominated in this series, an observation made by other local ${ }^{21}$ and international studies. ${ }^{17,18,24}$ Other studies, however, have shown a predominance of AKAs. ${ }^{7,19}$ The most likely explanation for this in the present series is that patients tend to present to the hospital with foot sepsis before it extends proximally, thus reducing the risk that an AKA will be required. Furthermore, surgeons tend to amputate distally to enhance functional activity which inadvertently leads to other higher amputations, if the pathological process is not halted by the first amputation. ${ }^{24}$ The higher frequency of multiple amputations per patient among diabetic amputees in this series supports this theory.

One of the main goals of rehabilitation for amputees is to gain optimal physical freedom, which necessitates a multidisciplinary team (MDT) approach, including physiotherapists, surgeons, orthotists, podiatrists, social workers, psychologists, and nurses specialising in amputation care..$^{10}$ Whereas a dedicated multidisciplinary amputation clinic is ideal to provide the optimal management and rehabilitation of amputees, unfortunately, no such clinic exists at this institution except for a combined physiotherapy/prosthetist clinic that takes place twice a month. In the current study, only $17.6 \%$ of patients were referred to physiotherapy following amputation. As with the previous local study ${ }^{10}$ from the same institution, the referral to physiotherapy remains very low. Further, of the small number of amputees referred to physiotherapy, $38.5 \%$ were non-compliant with the referral to physiotherapy and did not keep their appointments. Other barriers to proper amputee rehabilitation in the African context include poor healthcareseeking behaviour of the community dwellers, patronage of traditional health professionals, and poor referral practices by the health workers, as well as perceptual and financial obstacles to physiotherapy attendance. ${ }^{27,28}$ For these reasons, there is a lack of awareness amongst amputees and healthcare workers about the benefits of physiotherapy and/or barriers to meaningful integration of physiotherapy into their management at this facility and in other African settings.

Evidence suggests that the development of MDTs is one way to enhance the level of care by improving communication between referring clinicians, leading to a better interdisciplinary rehabilitation pathway. ${ }^{29}$ We would like to strongly emphasise to the authorities that the introduction of MDTs for the management and rehabilitation of amputees is crucial at Addington Hospital and the Durban metropolis where inter-hospital referral logistics and provision of awareness programmes to amputees could be integrated into care pathways to provide a more holistic amputee rehabilitation programme.

Despite the small number of patients (91) referred to physiotherapy, 87 were mobile by the end of their rehabilitation visits with at least an assistive device (95.6\%), which compares favourably to the $85 \%$ noted in our previous study. ${ }^{10}$ Notably, only $8 \%$ were fitted with a prosthesis following LEA compared to $70-92 \%$ reported in the international literature. ${ }^{9,30}$ Again, since the numbers of patients referred to and attending physiotherapy were minimal, the results cannot be generalisable to all patients with amputations in our setting.

A study by Cox et al. reported that patients with BKAs demonstrated higher functional independence postrehabilitation than AKAs. ${ }^{31}$ In the present study, patients with BKAs and AKAs were mobile with either crutches and prosthesis or walking frames and were almost similar at $96 \%$ and $93 \%$, respectively. It is gratifying to note that only a small number $(n=4)$ of patients who underwent different levels of amputations were wheelchair users. In this study, we did not record the symptom of phantom limb pain that occurs to some degree in $50-80 \%$ of all amputees. This complication adds challenges to the success of physical rehabilitation and one that needs to be factored into quality improvement studies going forward. . $^{30,31}$

We share Brown and Attinger's view that when counselling patients regarding limb salvage and amputation, their functional needs, goals, and the condition of their extremity should be considered, and that the goal should be to maximise their quality of life. ${ }^{32}$ Since LEA results in a profound change in a patient's life, one of the main objectives contributing to enhanced quality of life is social reintegration and systemic social support. ${ }^{33}$ Social integration is defined as an objective state relating to the number of contacts and interactions between persons and their wider social network. 
Systemic social support is the support provided by society to patients with impairment and handicap. ${ }^{33}$ Thus, low levels of social integration are associated with negative health outcomes. ${ }^{33}$ Poor participation in a rehabilitation programme designed for amputee reintegration into the socioeconomic environment presents significant challenges to the amputee functioning optimally and independently in the social and economic domains. This is specifically relevant in our setting since the majority of our patients already have challenging socioeconomic circumstances.

\section{Conclusion}

Despite the retrospective nature of this study and the lack of some key data points, this study at a single regional hospital does provide a valuable five-year window of insight. The majority of amputations were related to diabetic foot disorders. These individuals have more minor foot amputations and multiple amputations than non-diabetics. We have also shown glaring deficiencies in the system. These are poor referrals to physiotherapy services and poor adherence to the rehabilitation programme which functions well for those who attend.

These factors represent a system failure and require further analysis to plan for quality improvement. We believe that the first step, essential to improve patient outcomes, should be the introduction of a specialist MDT for amputees at Addington Hospital. Further interventions to improve DM management and foot care in the community and define care pathways should be explored.

\section{Acknowledgements}

Statistician - Mrs Catherine Connolly - University of KwaZulu Natal, Howard College, Discipline of Public Health Medicine.

\section{Conflict of interest}

The authors declare no conflict of interest.

\section{Funding source}

None.

\section{Ethical approval}

Regulatory approvals for the study from the Biomedical Research Ethics Committee of the University of KwaZuluNatal (BREC 236/19), the provincial Department of Health (KZ_201909_034).

\section{ORCID}

P Manickum (iD https://orcid.org/0000-0003-0398-0748 SS Ramklass (iD https://orcid.org/0000-0003-0451-8942 TE Madiba (iD https://orcid.org/0000-0002-0155-9143

\section{REFERENCES}

1. Al Agha R, Muneer H, AlQaseer A, Ismaeel T, Badr O. Major lower limb amputation: causes, characteristics and complications. Bahrain Med Bull. 2017;39(3):159-61.

2. Moxey P, Gogalniceanu P, Hinchliffe R, et al. Lower extremity amputations - a review of global variability in incidence. Diabet Med. 2011;28(10):1144-53.

3. Raghav A, Khan Z, Labala R, et al. Financial burden of diabetic foot ulcers to world - a progressive topic to discuss always. Ther Adv Endocrinol Metab. 2018;9(9):29-31.
4. Behrendt C-A, Sigvant B, Szeberin Z, et al. International variations in amputation practice - a VASCUNET report. Eur J Vasc Endovasc Surg. 2018;56(3):391-9.

5. Agha SA, Usman G, Agha MA, et al. Influence of sociodemographic factors on knowledge and practice of proper diabetic foot care. Khyber Medical University Journal. 2014;6(1):9-13.

6. Holman N, Young R, Jeffcoate W. Variation of the recorded incidence of lower limb amputation in England. Diabetologia. 2012;55:1919-25.

7. Unnikrishnan E, Rollands R, Parambil SM. Epidemiology of major limb amputations - a cross sectional study from a South Indian tertiary care hospital. Int Surg J. 2017;4(5):1642-6.

8. Chalya PL, Mabula JB, Dass RM, et al. Major limb amputations - tertiary hospital experience in northwestern Tanzania. J Orthop Surg Res. 2012;7(1):1-6.

9. Chamlian TR. Use of prostheses in lower limb amputee patients due to peripheral arterial disease. Einstein (São Paulo). 2014;12(4):440-6.

10. Manickum P, Ramklass S, Madiba T. A five-year audit of lower limb amputations below the knee and rehabilitation outcomes - the Durban experience. Journal of Endocrinology, Metabolism and Diabetes of South Africa. 2019;24(2):41-5.

11. Chimere-Dan O. Population policy in South Africa. Stud Fam Plann. 1993;24(1):31-9.

12. Encyclopedia Britannica. Coloured: Encyclopedia Britannica Inc; 2016. Available from: https://www.britannica.com/topic/ Coloured. Accessed 14 Mar 2020.

13. Goddard N, Brueton R. Orthopaedics and trauma: amputations. In: Novell R, Baker D, Goddard N, Kirk R, editors. Kirks general surgical operations. 6th ed. Churchill Livingston (Elsevier): Edinburgh; 2013. ch 30. p. 471-80.

14. Molina CS, Faulk J. Lower extremity amputation. Treasure Island (FL): StatPearls Publishing; 2019. Available from: https://europepmc.org/books/NBK546594;jsessionid=2CBD5 4F9A4B200C0E9272CB9E1C06209. Accessed 29 Nov 2019.

15. Godlwana L, Nadasan T, Puckree T. Global trends in incidence of lower limb amputation - a review of the literature. S Afr J Physiother. 2008;64(1):8-12.

16. Mtshali S, Mahomed O. Patient-level predictors of diabetesrelated lower extremity amputations at a quaternary hospital in South Africa. PloS One. 2020;15(10):1-9.

17. Kayssi A, De Mestral C, Forbes TL, Roche-Nagel G. A Canadian population-based description of the indications for lower-extremity amputations and outcomes. Can J Surg. 2016;59(2):99-106.

18. Odatuwa-Omagbemi DO. Extremity amputation in Warri, South-South Nigeria. J West Afr Coll Surg. 2012;2(1):14-24.

19. Dunbar G, Hellenberg D, Levitt N. Diabetes mellitus and nontraumatic lower extremity amputations in four public sector hospitals in Cape Town, South Africa, during 2009 and 2010. S Afr Med J. 2015;105(12):1053-6.

20. Awori K, Atinga J. Lower limb amputations at the Kenyatta National Hospital, Nairobi. East Afr Med J. 2007;84(3):121-6.

21. Cheddie S, Manneh CG, Pillay B. Spectrum of disease and outcome of primary amputation for diabetic foot sepsis in rural KwaZulu-Natal. S Afr J Surg. 2018;56(3):16-9.

22. International Diabetes Federation. IDF Diabetes Atlas. Brussels, Belgium: 2017. Available from: https://www.idf.org. Accessed 16 Feb 2019.

23. Desalu O, Salawu F, Jimoh A, et al. Diabetic foot care - self reported knowledge and practice among patients 
attending three tertiary hospitals in Nigeria. Ghana Med J. 2011;45(2):60-5.

24. Pooja GD, Sangeeta L. Prevalence and aetiology of amputation in Kolkata, India - retrospective analysis. Hong Kong Physiother J. 2013;31(1):36-40.

25. Coxon JP, Gallen IW. Laterality of lower limb amputation in diabetic patients - retrospective audit. $\mathrm{Br}$ Med J. 1999;318(7180):367.

26. De Asha AR, Buckley JG. The effects of laterality on obstacle crossing performance in unilateral trans-tibial amputees. Clin Biomech. 2015;30(4):343-6.

27. Igwesi-Chidobe C. Obstacles to obtaining optimal physiotherapy services in a rural community in southeastern Nigeria. Rehabil Res Pract. 2012;2012:1-8.

28. Chen A, Koh YT, Leong S, et al. Post community hospital discharge rehabilitation attendance: Self-perceived barriers and participation over time. Ann Acad Med Singap. 2014;43:136-44.
29. Ennion L, Rhoda A. Roles and challenges of the multidisciplinary team involved in prosthetic rehabilitation, in a rural district in South Africa. J Multidiscip Healthc. 2016;9:565-73.

30. Vetrayan J, Ghafar NBA, Paulraj SJPV, Murad MS. Occupational performance role and satisfaction among lower limb amputees with different adaptive devices usage. Procedia Soc Behav Sci. 2016;222:432-41.

31. Cox PSL, Williams SKP, Weaver S. Life after lower extremity amputation in diabetics. West Indian Med J. 2011;60(5):53640.

32. Brown BJ, Attinger CE. The below-knee amputation: to amputate or palliate? Adv Wound Care. 2013;2(1):30-5.

33. Hawkins AT, Pallangyo AJ, Herman AM, et al. The effect of social integration on outcomes after major lower extremity amputation. J Vasc Surg. 2016;63(1):154-62. 